\title{
Application of acoustic-electric interaction for neuro-muscular activity mapping: A review
}

\author{
Thordur Helgason (1,2), Kristin Inga Gunnlaugsdottir $(1,2,3,4)$
}

(1) Reykjavik University, Reykjavik, Iceland, (2) Landspítali-University Hospital, Reykjavík, Iceland, (3) University of Lübeck, Lübeck, Germany, (4) University of Applied Sciences, Lübeck, Germany

\begin{abstract}
Acousto-electric interaction signal (AEI signal) resulting from interaction of acoustic pressure wave and electrical current field has received recent attention in biomedical field for detection and registration of bioelectrical current. The signal is of very small value and brings about several challenges when detecting it. Several observations on the AEI signal have been done in saline solution and on nerves and tissues under controlled condition that give optimistic indication for its utilization. Ultrasound Current Source Density Imaging (UCSDI) has been introduced, that uses the AEI signal to image the current distribution. This review provides an overview of the investigations on the AEI signal and USCDI imaging that has been made, their results and several considerations on the limitations and future possibilities on using the acousto-electric interaction signal.
\end{abstract}

Key Words: Acousto-electric interaction signal, Ultrasound Current Source Density Imaging, tissues, muscles

\begin{abstract}
Acousto-electric signal is a result of interaction between pressure and current. As pressure wave propagates through a medium, it changes its conductivity locally. It causes an increase in conductivity when the pressure falls and a decrease when the pressure rises. Consequently if electrical current is flowing through the medium potential changes will take place.

Periodical change in pressure is produced by an ultrasound wave traveling through a conductive medium. This in turn modifies the conductivity of the medium. This is called the acousto-electric effect and can be described by the Eq. 1:
\end{abstract}

$$
\frac{\Delta \sigma}{\sigma}=K_{I} \Delta P
$$

where $\mathrm{K}_{\mathrm{I}}$ is the interaction coefficient characteristic for the medium, $\sigma$ is the conductivity, $\Delta \sigma$ conductivity change due to the ultrasound wave and $\Delta \mathrm{P}$ the pressure change. If an electrical current is flowing through the medium at the same time a periodical voltage change will take place due to the change in conductivity. The resulting signal is called the aousto-electric interaction signal (AEI signal). Its origin is at the location of the pressure changes. This principle is visualized in Figure 1.
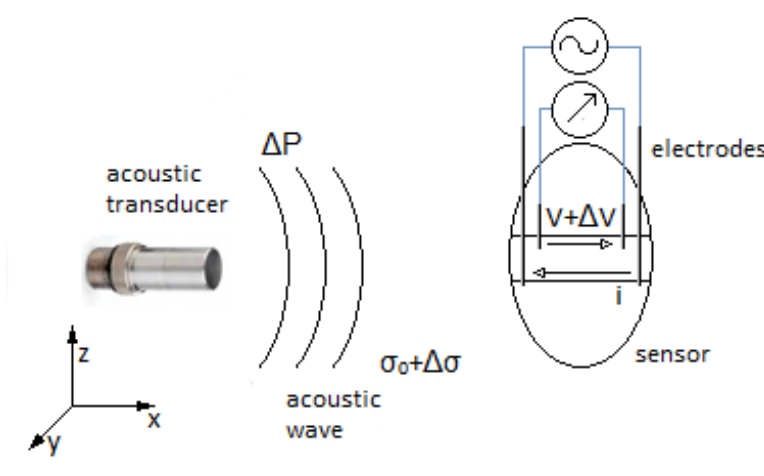

Fig 1. The principle of acousto-electric interaction signal. The pressure wave $(\triangle P)$ causes a local change in conductivity $(\Delta \sigma)$ of the solution. When electrical current (i) flows between two electrodes, perpendicular to the propagation direction of the acoustic wave, it generates electrical field within specific area. Operating simultaneously, they give a rise to a signal generation $(\Delta V)$, measured between two recording electrodes, that is both proportional to the pressure change and the current flowing. 


\section{Electron microscopy: from 2D to 3D muscle images}

Eur J Trans Myol - Basic Appl Myol 2014; 24 (4): 261-267

Using a focused ultrasound wave the strongest AEI signal will be generated in the focal point. The signal can be described by the Eq. $2:^{2}$

$$
V(t)=\iiint_{\text {medium }}\left(J^{L} \bullet J^{I}\right)\left(\sigma_{0}-K_{I} \sigma_{0} \Delta P\right) d x d y d z
$$

$\mathbf{J}^{\mathrm{L}}$ is the lead field of the recording electrodes and $\mathbf{J}^{\mathrm{I}}$ the distributed current source and $\mathrm{V}$ is the signal detected by electrodes. This equation can be rewritten:

$$
\begin{aligned}
& V(t)=\underbrace{\iiint_{\text {medium }}\left(J^{L} \bullet J^{I}\right) \sigma_{0} d x d y d z}_{\mathrm{V}^{\mathrm{LF}}} \\
& +\iiint_{\text {medium }}\left(J^{L} \bullet J^{I}\right)\left(-K_{I} \sigma_{0} \Delta P\right) d x d y d z
\end{aligned}
$$

The first term is a low frequency part of the injected current but the second term is the high frequency AEI signal. If the current density is constant, this frequency is the frequency of the ultrasound wave. The AEI signal can easily be filtered from other signals in living tissue, for example in muscle, as no natural signal has the frequency of an ultrasound wave.

Even though acousto-electrical effect has been observed since in the $1940 \mathrm{~s},{ }^{3}$ it has only recently received interest in the biomedical field. As it is quite recent, relatively little has been written so far about it in this field, nevertheless giving some positive indications about the efficiency of its use in biomedical applications. In the late 1990s a group of French academics renewed the interest in acosto-electrical effect when they started to investigate and observe the AEI signal in a saline solution. ${ }^{1,3-5}$ About a decade later a group from Canada also performed observations on it. $^{6}$ Subsequently Zhang and Wang used the acoustoelectrical effect in an experiment for imaging the electric impedance properties of biological tissues in acousto-electric tomography. ${ }^{7}$ Influential in the research of acousto-electric interaction signal in the recent years, has been a group of American researchers at the University of Michigan, that have performed several investigations on the AEI signal and the possibility of using the signal to map biological current. ${ }^{2,8-17}$ Several more studies have been performed for further investigations and improvements of measurements and setup. ${ }^{18}$ Also our group has made some investigations and come up with a potentially useful application for denervated muscles. ${ }^{19}$

\section{Measurements in saline solution}

\section{Acousto-electric interaction signal}

All of the observations of acousto-electric interaction signal have been performed in saline solution. Saline solution has similar composition as blood and extracellular fluid, containing approximately $0.9 \%$ $\mathrm{NaCl}$. Relatively simple measurement setup can be created, containing transducer transmitting a pressure wave, current generator and voltage recording. The dilemma is to control relevant variables and condition the signal, as the signal is of low magnitude.

This principle of setup is fundamental in the observations of acousto-electrical effect and is similar to all experiments (see Figure 2), though the geometry, derivation and values of distinctive parameters may differ. In principle, the pulser-receiver transmits an electrical pulse to the transducer that generates corresponding pressure wave. As the pressure wave propagates in the medium, the conductivity of the medium changes locally in the direction of the wave. A measurement cell, containing current injecting electrodes and recording electrodes is placed at the focal point of the transducer, where the maximum pressure occurs. The outer pair of electrodes injects the current, while the recording electrodes detecting the AEI signal are located within the current density field. These parameters may differ, as it is also possible to detect the AEI signal with only one recording electrode and a ground reference electrode. The cell has a defined geometry, which helps to control and evaluate the current density. The recorded signal is amplified before it is visualized on an oscilloscope. Hydrophone is often used to measure the pressure amplitude of the acoustic wave.

Jossinet, Lavandier and Cathignol investigated anew the acousto-electrical interaction signal at the end of last century. They reviewed the phenomena involved the generation of the AEI signal., ${ }^{3,4}$ The changes in conductivity arises from variations in pressure and temperature. Pressure is the primary cause, bringing about adiabatic process of conversion of the mechanical energy of pressure wave to heat, thus changing the temperature. This procedure is adiabatic process, as the time for heat transfer to take place is not enough within the period of acoustic wave. This procedure affects the properties of the solution in three ways: the bulk compressibility, the ionic mobility and the dissociation equilibrium. Bulk compressibility is caused by changes in the specific volume of the solutions and brings about changes in molarity; ionic mobility is affected by the change in viscosity; and dissociation coefficient of partially dissociated species varies both with temperature and pressure. For strong electrolytes, the dissociation coefficient is equal to unity and the electrolytes are totally dissociated. For a $\mathrm{NaCl}$ solution, bulk compressibility is responsible for about $47 \%$ of the effect, $18 \%$ are due to the effect of pressure upon the change in viscosity and changes of ionic mobility against temperature accounts for the rest $35 \%$.

Special attention has to be paid measuring the AEI signal, as it is of small value. Jossinet et al. detected the acousto-electrical signal in saline solution, utilizing 


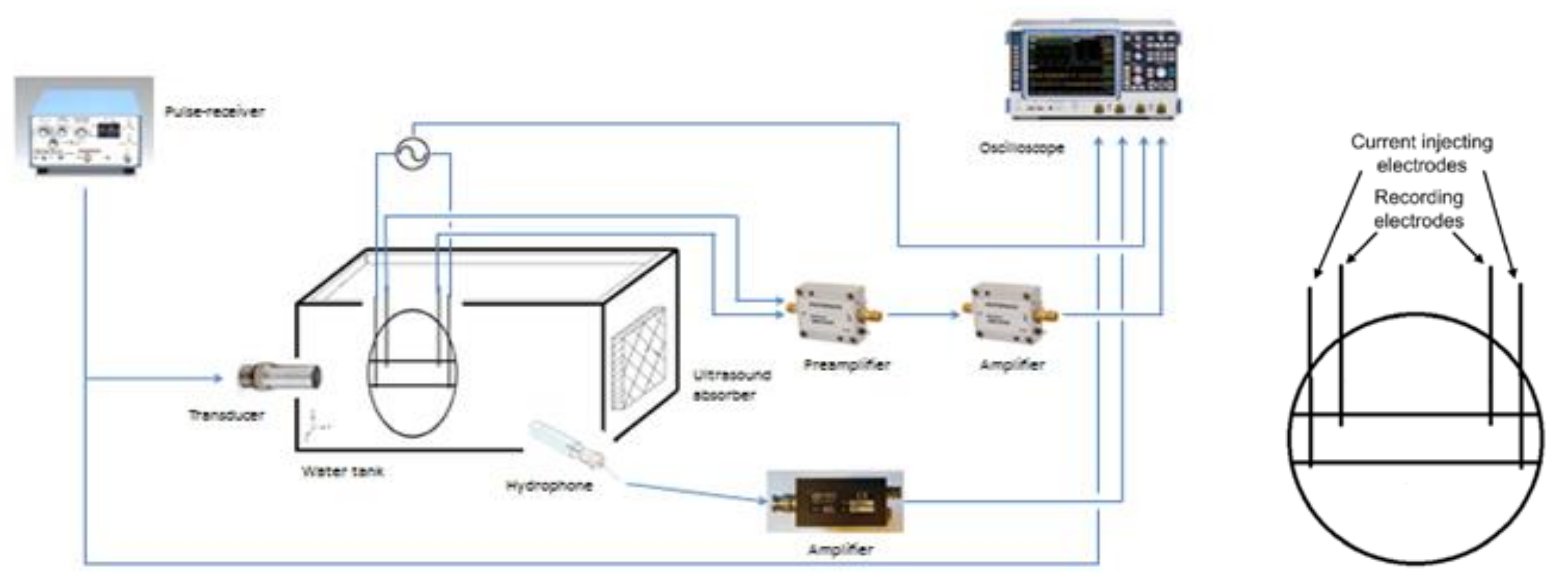

Fig 2. An example of experimental setup of measurement of acousto-electrical signal in saline solution, with more detailed view of the measurement cell. The recording electrodes of the measurement cell lay within the current density generated by the injecting current electrodes.

experimental setup comparable to the one described above. ${ }^{4}$ It was only several microvolts. A very small change in conductivity is caused by change in pressure, about $0.1 \%$ per $\mathrm{MPa}$ for $\mathrm{NaCl}$ solution, 1,5 which contributes to small AEI signal. Additionally, a sinusoidal pressure wave, including both positive and negative pressure peaks, produces opposite conductivity changes within one wavelength. Over larger distance, as the distance between the recording electrodes, the opposite changes of conductivity tend to neutralize the influence of each other on the local resistance, making the acousto-electrical signal smaller. The use of unipolar pulses tends to avoid this problem. ${ }^{5}$ The signal is also sensitive to increased measured impedance because of the summation of the medium impedance and electrode contact impedance, increasing the overall impedance. The nonuniform sensitive distribution of the electrodes also plays a role, as well as imperfections in geometry and uncertainties in estimation of the interaction coefficient $\mathrm{K}^{4,5}$ The acoustoelectric interaction constant $\mathrm{K}$ determines how much the electrical resistivity changes due to ultrasound pressure. $\mathrm{Li}$ et al. estimated the constant for different ionic salts and cardiac tissue. ${ }^{1,6} \mathrm{~K}$ appears to vary with concentration in a solution of many ions, such as $\mathrm{CuSO}_{4}$, because of the degree of dissociation of the ions in solution, whereas in monovalent salt, like $\mathrm{NaCl}$, no such difference is observed. From the seven hearts observed, $\mathrm{K}$ in the heart was measured to be $0.041 \pm 0.012 . \% / \mathrm{MPa}$. The constant between different hearts varied slightly, which could be because ofdistinctiveness between the hearts and tissue preparation, but the interaction constant did not differ much with varying temperature. The $\mathrm{K}$ in hearts is of similar value as in $0.9 \%$ saline $(0.034 \pm 0.003 . \% / \mathrm{MPa})$. That can be explained, asthe composition of the
$0.9 \% \mathrm{NaCl}$.

In order to evaluate the acoustic signal, the measured Ultrasonic Vibrational Potential (UVP) or Debye effect, has to be taken into account. Debye effect arises from periodical displacement of the ions due to pressure, causing unequal distribution of negative and positive charges and subsequently potential difference. Debye effect emerges without applied current and has to be subtracted from the measured detected voltage in order to receive the AEI signal. Theoretically in order to get the greatest AEI signal, the current should be perpendicular to the ultrasound propagation. However at $90^{\circ}$ the Debye effect component is so small and hard to detect, that it would most certainly influence the measured signal and induce some error. It is therefore better to measure at a smaller angle, where it is easier to detect the Debye effect. ${ }^{1}$

The relationship between the AEI signal and current and pressure respectively has been investigated. Higher signal follows increased pressure and increased current density. ${ }^{3,5}$ The effect of pressure and current on the signal has been measured, by keeping one parameter constant while the other one varied. ${ }^{1}$ The signal is linearly proportional to both, with an interaction factor of $5.3 \mu \mathrm{V} \mathrm{mA} \mathrm{mPa}^{-1}$ between the voltage, pressure and current. The measured AEI signal in saline solution was in the order of several dozen microvolts under controlled condition. Gendron, Guardo and Bertrand achieved an AEI signal in the order of hundreds of microvolts in their experimental setup, after eliminating the Debye effect by subtracting the AEI signal derived at the negative current peak from the one derived at the positive peak. ${ }^{6}$ Their setup resembled the previously used as described above. Additionally, it was observed that the signal was not directly proportional to the electric signal driving the transducer and a certain threshold was also needed to be reached in order to detect the AEI signal. This could 


\section{Electron microscopy: from 2D to 3D muscle images}

Eur J Trans Myol - Basic Appl Myol 2014; 24 (4): 261-267

be a result of cavitation that arises in the decompression phase of the ultrasound. These bubbles affect the conductivity of the solution, influence thus the AEI signal and can lead to incorrect measurement of the signal. Special attention should be paid in order to avoid cavitation, by using pressure amplitude below cavitation formation threshold or by using unipolar pulses.

\section{Ultrasound Current Source Density Imaging}

The acousto-electrical interaction signal can be reconstructed and used in medical imaging. Zhang and Wang developed the idea of acousto-electric tomography (AET) to image electric impedance. ${ }^{7}$ The measurement was performed in a bath of mineral oil, which promotes acoustic coupling and electrical isolation. The difference in conductivity of rubber in saline solution could clearly be seen in 2D and its size be identified. Fat on a chicken breast tissue was also imaged. As the tissue is not homogenous, like saline, several discontinuities were observed in the image. The spatial resolution of acousto-electric tomography was of $2.3 \mathrm{~mm}$, with a good contrast. The resolution was limited by the focal size of the ultrasound. Comparing this to electric impedance tomography (EIT), AET had better resolution and simpler process.

The team from Michigan has been focusing on electrical mapping using acousto-electric effect, a technique called Ultrasound Current Source Density Imaging (UCSDI). Electrical mapping has mainly been done in $2 \mathrm{D}$ but can be made in $3 \mathrm{D}$ by using the same procedure. In one of their experiments, a current distribution in two dimensions was reconstructed. ${ }^{8} \mathrm{~A}$ current, resembling the current of the heart, with peak current density of $8 \mathrm{~mA} / \mathrm{cm}^{2}$ was injected by a pair of electrodes into a chamber filled with saline solution. The reconstructed signal represents relatively well the simulated ECG signal, though the some parts of the high frequency were missing. An accuracy of approximately $1 \pm 2 \mathrm{~mm}$ was achieved in reconstruction of a 2D dipole field in a saline solution, by assuming that the current is coherent within a beamwidth. ${ }^{9}$ Likewise a current source and sink were successfully located in 2D, with the precision accomplished to be within $1 \mathrm{~mm}$, and corresponded well with simulated data. ${ }^{2}$ With the resistivity distribution previously estimated, the accuracy was limited by the sampling interval, which was of $2.2 \mathrm{~mm}$. This method can also be used for time dependent volume imaging, as shown using alternative current for depicting dipole field. ${ }^{10}$ The reconstruction was made in a saline solution, with ultrasound transducer scanned in a plane. At each time interval electrodes together with a ground reference depicted an image. Timevarying image of the current distribution was obtained, though the slow scan time necessary to obtain the4D data introduces some limitations, but more than an hour was required to obtain the image. 4D UCSDI simulation of a dipole field was developed for comparison and validation of the AEI signal. ${ }^{11}$ Multichannel UCSDI could potentially improve the mapping and achieve more detailed information about the internal current distribution of tissues.

UCSDI has a high spatial resolution and sensitivity. The resolution is determined by the ultrasound beam and sampling interval., ${ }^{2,9}$ The frequency and the shape of the beam also matters. ${ }^{12}$ By using higher ultrasound bandwidth, the spatial resolution was improved, but on the cost of sensitivity. Likewise, chirp excitation of the ultrasound improved the sensitivity, compared to square wave. Yang et al. from University of Minnesota proposed unipolar pulses to the ultrasound in order to reconstruct an image of current density distribution. ${ }^{18}$ By using unipolar pulses instead of bipolar, the generated AEI signal turns out to be stronger, with an increase of more than 250 times, when using pressure amplitude of $1 \mathrm{MPa}$. According to Yang, the unipolar pulses give thus a better estimation of the current density distribution, but the setup needs at least three recording electrodes. The AEI signal detected by using unipolar pulse, showed more reliable variation within the measurement area, as it is not as sensitive to boundaries where strong current gradient exists, but is instead more sensitive to variation within the imaged object. It has stable performance for different parameter setups and noise is minimal. Further optimization of the ultrasound parameters, could improve the sensitivity and imaging of bioelectrical current even more.

The geometry can be co-registered by the traditional Bscan ultrasound, along with the AEI signal recording, by using simultaneously the pulse echo signal generated by the ultrasound transducer.. ${ }^{9}$ This procedure could offer valuable improvements compared to existing examining methods, as it offers anatomical image during registration of the electrical activity within the body. This could offer possibilities in surgical guidance and during treatment of various diseases. Conventional methods use often computed tomography (CT) or magnetic resonance image (MRI) to map the anatomy and give a static image prior to intervention. But during the procedure, it is often not possible to review the circumstances. The registration error between the CT and/or MRI to the electroanatomical mapping has been reported to be about $2-10 \mathrm{~cm}^{2}$ This method could facilitate corrective procedures for cardiac and neural abnormalities. ${ }^{17,15,11}$ These experiments use measurement setups in a saline solution under controlled condition. This demonstrates the optimum condition for detecting the small AEI signal. The geometry is controlled, the solution is homogenous and relatively little disturbances encountered. Furthermore, it is assumed that the conductivitity distribution is assumed to be known in these setups. Within the body the resitivity distribution vary and is gernerally not know beforehand, but it 


\section{Electron microscopy: from 2D to 3D muscle images}

Eur J Trans Myol - Basic Appl Myol 2014; 24 (4): 261-267

could be assumed using the images from electrical impedance tomography or tissue segmentation using CT or MRI. ${ }^{18}$ Several difficulties are met in recording the AEI signal for the UCSDI. The detected AEI signal has to be appropriately recorded, filtered and conditioned. Also, the geometry brings about limitation, as it is not possible to register near to the injecting current wires. ${ }^{2}$ Also tThe scan time and acquisition rate is limited by scanning of the transducer. In theory, UCSDI should be able to perform as fast as traditional pulse-echo ultrasound, or even faster because it only demands one-way propagation of the acoustic wave and not returning echo as pulse-echo ultrasound. ${ }^{11}$

\section{Observations in nerves}

While most of the observations have been focused on the acousto-electric effect itself, relatively few observations have been made on these effects on biological nerves or tissues so far. The team from the University of Michigan has made observation on AEI in sciatic nerve of a rat, lobster nerve cord and tissues from chicken. Experimental system was well controlled. A piece of the nerve or the tissue was cut, isolated and immersed in mineral oil that serves as acoustic coupling and electrical insulation. Specific geometrical dimension of both the investigated objects and the measurement setup help to register the AEI signal and offers relatively simple software model.

Acousto-electrical effect was used to image current distribution in a sciatic nerve that was obtained fresh from a rat. ${ }^{13}$ The nerve was isolated, immersed in mineral oil and placed on pairs of electrodes that either wise injected current or recorded the voltage within the current density field. In this specific geometry it was possible to determine electrical current spatially and temporally. Simultaneously a pulse-echo image was registered, that matched to the AEI signals. The signal was proportional to the current density, which was itself in relation to signal to noise ratio.

Similar procedure was followed when imaging injected current in an abdominal segment of a fresh lobster nerve cord. ${ }^{14}$ Three-compartment chamber was used for this experiment, where the central part of the nerve was placed in middle chamber filled with mineral oil. The injecting electrodes were situated in the side chambers, giving electrical current through the nerve, while recording electrodes were located at the exterior of the nerve in the middle chamber. The AEI signal measured showed that the acoustoelectric signal was directly proportional to both the applied pressure and current density, where the focal pressure forms a slope of $23 \mu \mathrm{V} / \mathrm{MPa}$ at $75 \mathrm{~mA} / \mathrm{cm}^{2}$, whereas the current density forms a slope of $0.7 \mu \mathrm{V} / \mathrm{mA} \mathrm{cm} \mathrm{cm}^{2}$ at $2 \mathrm{MPa}$. The current density and the pressure were at physiological values, where the current density was less than $10 \mathrm{~mA} / \mathrm{cm}^{2}$ and pressure in the range of clinical ultrasound imaging.
Finally, two tissues from turkey were investigated simultaneously. ${ }^{15}$ Two distinctive slices from a tissue of a turkey were initially placed in saline solution before inserted into a chamber filled with mineral oil at different distance from the transducer. The current was injected to the slices in opposite directions and recording electrodes connected to each and subsequently to a differential amplifier. The slices were located very close to each other, separated by a thin film of $0.2 \mathrm{~mm}$. The recorded AE images appeared to be identical to the real tissues, in size and location, and the tissues could be successfully separated. The AEI signal was proportional to both pressure and current, with the detection levels in the order of 1 $\mathrm{mA} / \mathrm{cm}^{2}$ at $258 \mathrm{kPa}$. The magnitude and direction of the current, the location of the current field within the ultrasound field and the geometry play all part in the construction of the image and sensitivity is determined by the diameter of the acoustic beam.

\section{Observations in muscles}

Even fewer observations have been made on acoustoelectric interaction signal in muscles than with nerves and tissues. At the University of Michigan an experiment has though been performed where a biopotential of a fresh rabbit heart was measured. The preparation and procedure resembled with previous experiments of the nerves and tissues, where the heart was decoupled, isolated and placed in specific holder that specifies the outlay of the heart.

UCSDI imaging was used to map biopotential in a fresh heart of a rabbit. ${ }^{17}$ The heart was injected with the excitation-contraction decoupler BDM to suppress the motion, avoiding motion artifacts. The heart was isolated and placed in a predetermined way in a tank that was surrounded by a bigger tank filled with deionized water. Four stimulating electrodes were placed in the heart; two in the apax and another pairin the right atrium. The recording electrodes were inserted in the left ventricle along the long axis of the heart approximately. Under this controlled condition, it was possible to detect the spreading activation wave, spatially and temporally, that had a propagation velocity estimated to be $0.25 \pm 0.05 \mathrm{~mm} / \mathrm{ms}$. The detection levels were in the order of $1 \mathrm{~mA} / \mathrm{cm}^{2}$ at 258 $\mathrm{kPa}$. This velocity is comparable to the results obtained from electrocardiogram (ECG). A limitation of this study was the hardware limitations that could not acquire all the electrodes simultaneously.

Gudjonsdottir et al. has derivate that it is possible to use AEI signal to map the current distribution in denervated thigh muscle. ${ }^{19}$ The AEI signal that would be detected is of measurable dimensions and frequency that is easy to filter out. This can be practical during functional electrical stimulation (FES) on the thigh, to ensure that the whole muscle is stimulated during the treatment and estimating about the efficiency of the treatment. 


\section{Electron microscopy: from 2D to 3D muscle images}

Eur J Trans Myol - Basic Appl Myol 2014; 24 (4): 261-267

\section{Conclusions and discussion}

These researches on the acousto-electrical interaction signal have shown that it is possible to detect the acousto-electric effect and reconstruct ultrasound current source density image (UCSDI) to model the distribution of current density. So far, the acoustoelectrical effect or the AEI signal has only been observed in saline solution under controlled condition. It has appeared to be sensitive enough to detect biological current, by using safe levels of clinical ultrasound. It should be born in mind that the AEI signal is measured under optimal condition, where the geometry is controlled and defined in a clear way. Unlike physiological tissues, saline solution is homogenous and minimal disturbances are encountered. In physiological condition, the conductivity distribution and sensitivity distribution are nonhomogenous and unknown. As the AEI signal is of very low amplitude, this could introduce specific problems when encountering it within biological tissues. Critical is to use safe amount of current, so the patient would not perceive it and ultrasound within clinical limits, to avoid destruction of some tissues. Also cavitation has to be avoided, as it can cause tissue damage. Higher ultrasound frequencies do not penetrate as deep as lower one, but have better resolution. More research is needed in order to get sufficient in depth information about the signal and its possible usage.

A big advantage of UCSDI is that it is noninvasive and would thus not affect or destroy organs. UCSDI has a high spatial and temporal resolution, which is limited by the ultrasound beam geometry, pulse length and data recording. So far, it has mainly been recorded in 2D, but 3D dimensional mapping should also be possible, even though scan time and acquisition rate are still really time consuming. Optimizations are still required before it will be possible to utilize it.This method could offer valuable improvements, compared to existing examining methods. It has the potential to offer anatomical image simultaneously with registration of electrical activity within the body. This could be useful during treatment or surgical intervention UCSDI could be used to record and register in the treatment of cardiac, muscular and neural abnormalities.

Few observations have been reported for nerves and tissues and only cardiac tissue of rabbits has been investigated in vitro. The specific dimensions of the tissues and the isolation from other tissues with the mineral oil make it easier to detect the acoustoelectrical effect, than it would in in vitro. It can though be expected that the AEI signal can be measured in skeletal muscles, though many more observations on the skeletal muscle are still needed. The AEI signal is of small amplitude and is sensitive to disturbances, noise and other currents, especially when the medium has larger dimension than the ultrasound wave length as within the human body. Precise measurement devices, performances and processing methods are needed in order to capture the desired signal and its direction. It is a promising field, that could lead to a lot of possibilities for use in medicine and the biological field.

\section{Acknowledgements}

This work was supported by the Science Found of Landspitali - University Hospital.

\section{Corresponding Author}

Thordur Helgason, Reykjavik University/Uni. Hospital, Menntavegur 1, 101 Reykjavík, Iceland. Tel. +3458245737

E-mail: thordur@landspitali.is

Co-Author E-mail: kristin.gunnlaugsdottir@gmail.com

\section{References}

1. 1. Lavandier $\mathrm{B}$, Jossinet $\mathrm{J}$, Cathignol $\mathrm{D}$. Experimental measurement of the acousto-electric interaction signal in saline solution. Ultrasonics 2000;38:929-36.

2. Olafsson R, Witte RS, Huang S-W, O’Donnell M. Ultrasound Current Source Density Imaging. IEEE Trans Biomed Eng 2008;55:1840-8.

3. Jossinet J, Lavandier B, Cathignol D. The phemenology of acousto-electric interaction signal in aqueous solution of electrolytes. Ultrasonics 1998;36:607-13.

4. Jossinet J, Lavandier B, Cathignol D. Impedance modulation by pulsed ultrasound. Ann N Y Acad Sci 1999;873:396-407.

5. Lavandier B, Jossinet J, Cathignol D. Quantitative assessment of ultrasound-induced resistance change in saline solution. Med Biol Eng Comput 2000;38:150-5.

6. Gendron M, Guardo R, Bertrand M. Experimental setup for developing acousto-electric interaction imaging. Conf Proc IEEE Eng Med Biol Soc 2009;2009:2279-83.

7. Zhang H, Wang LV. Acousto-electric tomography. Proc SPIE Photons Plus Ultrasound Imag Sens 2004;5320:145-9.

8. Olafsson R, Witte RS, Kim K, et al. Electric current mapping using the acousto-electric effect. Proc SPIE Med Imag Ultrason Imag Signal Process 2006;6147:614701-1-614701-11.

9. Olafsson R, Witte RS, O'Donnell M. Measurement of a 2D electric dipole field using the acousto-electric effect. Proc SPIE 6513 Med Imaging 2007 Ultrason Imaging Signal Process 2007;6513:5130S.

10. Wang ZH, Olafsson R, Ingram P, et al. Fourdimensional ultrasound current source density imaging of a dipole field. Appl Phys Lett 2011;99:113701-13. 


\section{Electron microscopy: from 2D to 3D muscle images}

Eur J Trans Myol - Basic Appl Myol 2014; 24 (4): 261-267

11. Wang Z, Witte RS. Simulation-based validation for four- dimensional multi-channel ultrasound current source density imaging. IEEE Trans Ultrason Ferroelectr Freq Control 2014;61:420-7.

12. Qin $Y$, Wang $Z$, Ingram $P$, et al. Optimizing frequency and pulse shape for ultrasound current source density imaging. Ultrason Ferroelectr Freq Control IEEE Trans On 2012;59:2149-55.

13. Olafsson R, Li Q, Wang Z, et al. Ultrasound current source density imaging of a time-varying current field in a multielectrode nerve chamber. Ultrason Symp IUS 2009 IEEE Int 2009;333-6.

14. Witte R, Olafsson R, Huang S-W, O'Donnell M. Imaging current flow in lobster nerve cord using the acoustoelectric effect. Appl Phys Lett 2007;90:163902.

15. Wang Z, Ingram $P$, Olafsson $R$, et al. Detection of multiple electrical sources in tissue using ultrasound current source density imaging. Proc SPIE Med Imaging 2010 2010;7629:76290H.
16. Li Q, Olafsson R, Ingram P, et al. Measuring the acoustoelectric interaction constant using ultrasound current source density imaging. Phys Med Biol 2012;57:5929-41.

17. Olafsson R, Witte RS, Jia C, et al. Cardiac activation mapping using ultrasound current source density imaging (UCSDI). IEEE Trans Ultrason Ferroelectr Freq Control 2009;56:56574.

18. Yang R, Li X, Liu J, He B. 3D current source density imaging based on the acoustoelectric effect: a simulation study using unipolar pulses. Phys Med Biol 2011;56:3825-42.

19. Gudjonsdottir B, Gargiulo P, Helgason T. Use of ultrasound current source density imaging (UCSDI) to monitor electrical stimulation of denervated muscles and fiber activity, some theoretical considerations. 10th Vienna Int Workshop Funct Electr Stimul 15th IFESS Annu Conf 2010;384-7. 